'Departamento de Ética del Colegio Médico de Chile A.G. Santiago, Chile. ${ }^{2}$ Centro de Bioética, Facultad de Medicina, Clínica Alemana Universidad del Desarrollo. Santiago, Chile.

${ }^{3}$ Departamento de Ciencias Neurológicas, Universidad de Chile. Santiago, Chile. ${ }^{4}$ División de Obstetricia y

Ginecología, Escuela de Medicina, Facultad de Medicina, Pontificia Universidad Católica de Chile. Santiago, Chile.

${ }^{5}$ Programa Cuidados Paliativos Hospital de Angol. Angol, Chile.

${ }^{6}$ Departamento de Medicina Interna, Universidad de Chile. Santiago, Chile.

${ }^{7}$ Departamento Jurídico, Colegio Médico de Chile A.G. Santiago, Chile.

8Programa de Genética Humana, ICBM, Facultad de Medicina, Universidad de Chile. Santiago, Chile. ${ }^{9}$ Departamento de Neuropediatría, Universidad de Valparaíso. Valparaíso, Chile.

${ }^{10}$ Directora Médica, Clínica Hospital del Profesor. Santiago, Chile. ${ }^{\text {aAbogado. }}$

Conflictos de interés: Ninguno que declarar. Este trabajo no obtuvo financiamiento.

Recibido el 14 de agosto de 2019, aceptado el 3 de febrero de 2020.

Correspondencia a: Sofía P. Salas Centro de Bioética, Facultad de Medicina, CAS-UDD. Avda. Las Condes 12.461, Torre 3, of 202. sofiasalas@udd.cl spsalas@gmail.com

\section{Argumentos éticos a favor y en contra de la participación del profesional médico en la muerte asistida. Análisis del Departamento de Ética del Colegio Médico de Chile}

\author{
SOFÍA P. SALAS ${ }^{1,2}$, RODRIGO A. SALINAS ${ }^{1,3}$, MAURICIO BESIO ${ }^{1,4}$, \\ CONSTANZA MICOLICH ${ }^{1,5}$, ANAMARÍA ARRIAGADA ${ }^{1,6}$, \\ ADELIO MISSERONI RADDATZ ${ }^{1,7, a}$, CARLOS Y. VALENZUELA ${ }^{1,8}$, \\ FERNANDO NOVOA ${ }^{1,9}$, GLADYS BÓRQUEZ ESTEFÓ ${ }^{1,10}$
}

\section{Ethical arguments for and against the participation of the medical profession in assisted death: analysis of the Ethics Department of the Chilean Medical Association}

The discussion of a bill that allows medically assisted death (MAD) in Chile, revived the debate about the ethics of this practice. The Department of Ethics of the Chilean Medical Association herein analyzes arguments in favor or against the participation of the medical profession in MAD. Among the main arguments against the participation of physicians in this practice are that MAD conflicts with the basic ethical principles of medical practice, that it is contrary to the purposes of medicine and that it could erode the patients' and society's confidence in physicians. The arguments in favor are related to physician's compassion and non-abandonment of patients during their illness, choosing palliative care and ushering them to the final instance. Additionally, there is social expectation that this practice will be carried out by trained physicians who can verify that the strict criteria established by the legislation are met, guarantee that it obeys to a repeated request of a fully capable patient, and who is able to deal with the complications of the procedure. In this document we aimed to represent the different perspectives about physicians' participation in MAD, offering arguments to colleagues and stimulating their participation in this important debate.

(Rev Med Chile 2020; 148: 542-547)

Key words: Ethics; Euthanasia; Palliative Care; Physician's Role; Suicide, Assisted.
$\mathrm{E}$ n enero de 2014, el presidente del Colegio Médico de Chile (COLMED) recibió una carta del Dr. Manuel Almeyda, de 89 años, quien aquejado de una enfermedad terminal, solicitaba al COLMED la creación de una comisión que estudiara la forma de poner término a la vida de las personas "que estén en una condición de

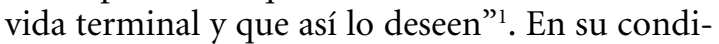
ción de médico y paciente, reflexionaba en torno al rol que le cabe a la profesión médica en terminar 
con la vida de un paciente que se encuentra en esa trágica condición, haciendo presente un problema moral que ha desafiado al ser humano por siglos, como es el de la legitimidad de terminar con la vida de una persona que experimenta un sufrimiento insoportable ${ }^{2}$. Tras negarse a ingerir alimentos y líquidos, Almeyda falleció sin ver acogida su petición.

El ingreso a discusión legislativa de un proyecto que establece el derecho a la eutanasia en Chile ${ }^{3}$, reavivó el debate respecto a la pertinencia ética de esta práctica. En síntesis, este proyecto propone permitir que enfermos terminales o que estén sufriendo de manera insoportable sin obtener alivio con las medidas que la medicina les ofrece, puedan solicitar terminar con su vida, ya sea mediante eutanasia, que consiste en la administración directa de una droga letal, o por suicidio médicamente asistido, donde se le prescriben los fármacos para que la persona acabe con su propia vida ${ }^{4,5}$. Ambas figuras corresponden a muerte médicamente asistida (MMA) y requieren de la participación de un médico para realizarlas.

Los argumentos a favor y en contra de la muerte asistida han sido ya expuestos por destacados expertos nacionales ${ }^{6-10}$. A propósito de la carta del Dr. Almeyda, como Departamento de Ética del COLMED nos pareció relevante analizar los argumentos éticos a favor y en contra de la participación del médico en este controvertido asunto. Para lograr un consenso, utilizamos el método deliberativo, lo que nos permitió evidenciar los valores en conflicto que subyacen a nuestras opiniones y juicios particulares, y que fundamentan nuestras argumentaciones.

\section{La muerte asistida a lo largo de la historia}

La participación del médico en la MMA se ha visto marcada por la influencia de la escuela hipocrática ${ }^{11} y$ por el predominio de las religiones judeocristianas en la conformación de la cultura y valores de Occidente. La escuela hipocrática prohibió a los médicos la administración de drogas mortales a sus pacientes, eliminando de su práctica una acción aceptada para escapar de los sufrimientos ocasionados por problemas de salud penosos e incurables ${ }^{2}$. A esto se sumó la condena que las religiones judeocristianas establecieron tanto al suicidio, en toda ocasión, como al homicidio, en la mayoría de los casos. Con la llegada del Renacimiento y de la Ilustración, algunos pensadores plantearon la legitimidad del auxilio a la muerte, esta vez como resultado de la autonomía del individuo llevada a la categoría de valor, o bien como atributo de sociedades ideales, en las que los enfermos incurables tenían acceso a acelerar sus muertes, aconsejados por sacerdotes o asistidos por sus médicos ${ }^{12}$. La introducción de valoraciones seculares, sumado al acceso a sustancias como la morfina y el cloroformo, permitieron el surgimiento de voces que proponían utilizarlas para terminar con la vida de enfermos terminales con sufrimiento insoportable, siendo un aspecto interesante en la discusión aquellos relacionados con la verdadera autonomía de quien se encuentra en estas trágicas circunstancias ${ }^{13}$. Los abusos del nazismo y la falta de adhesión a estas propuestas al interior de la profesión médica, impidieron que alcanzaran un estatus de legalidad hasta que Holanda, en 1985, despenalizó de facto la muerte provocada por médicos en pacientes terminales -legalizándola en 2002-, bajo estrictas condiciones $^{14,15}$. Posteriormente se legalizó la MMA en Bélgica, Luxemburgo, Canadá, Colombia y el estado de Victoria, en Australia, además de algunos estados norteamericanos ${ }^{16}$. Suiza, por su parte, despenalizó la asistencia al suicidio desde la primera mitad del siglo XX.

\section{Argumentos contrarios a la participación de médicos en la MMA}

La Asociación Médica Mundial (AMM) condena sin ambages la participación de médicos en estas acciones, por considerarlas contrarias a los objetivos de la medicina. En su reciente Declaración sobre Eutanasia y Suicidio Médicamente Asistido, señala que ningún médico puede ser obligado a participar en estas prácticas. Asimismo, reconoce que el médico que respeta el derecho básico del paciente a rechazar tratamientos médicos, no actúa de manera poco ética al no iniciar o suspender el cuidado médico, aun cuando de esto se derive la muerte del paciente ${ }^{17}$. Esta negativa a la participación de médicos en la MMA es compartida por numerosas asociaciones médicas, entre las cuales se encuentra el COLMED, cuyo Código de Ética señala: "El médico no podrá realizar acciones cuyo objetivo directo sea poner 
fin a la vida de un paciente bajo consideración alguna" $\left(\text { Art. } 9^{\circ}\right)^{18}$. Asimismo, la Sociedad Médica de Santiago argumentó que la eutanasia no es un acto que contribuya a ayudar a los pacientes, sean ellos terminales o no ${ }^{19}$.

Para comprender esta negativa, es necesario recordar que la labor primordial de los médicos ha sido siempre la de ayudar a los pacientes y aliviar su sufrimiento, por lo que provocarles la muerte suele ser considerado un fracaso de la medicina. Quienes argumentan en contra de la participación del médico en la MMA señalan que el sentimiento de compasión -que es el sentimiento de pena, ternura y de identificación ante los males que sufre el paciente-, nunca puede llegar a ser tan fuerte como para justificar estas acciones. La medicina ha logrado proveer a los pacientes de recursos eficaces para mitigar su sufrimiento que, sumados al desarrollo de los cuidados paliativos, permiten un abordaje integral del paciente y de su situación vital, sin perder de vista que existen aspectos personales, familiares, sociales o de orden espiritual, que van más allá de la responsabilidad del médico. Concordante con este pensamiento, si el médico participara acelerando la muerte de sus pacientes, actuaría solamente como un ejecutor de una muerte provocada por una sociedad incapaz de proporcionarles ayuda y alivio verdaderos.

Asimismo, quienes se oponen a la participación del médico en estas prácticas, aluden a que estas acciones no se encuentran comprendidas entre los "fines de la medicina" ${ }^{20}$, que, si bien contemplan el alivio del dolor y del sufrimiento causados por la enfermedad e idealmente que ocurra una muerte en paz, no implican el deber del médico de ocasionar la muerte de un paciente que lo solicite, sino más bien proveer los cuidados necesarios para alcanzar los fines señalados. De no ser así, se cree que esto podría erosionar la relación de confianza que debe existir entre el paciente y su médico, al asumir este el doble propósito de proveer salud y, excepcionalmente, de ocasionar la muerte ${ }^{21}$. Para otros detractores liberales, la participación del médico en estas prácticas no sería más que una expresión de la corriente medicalizadora de la vida y de la muerte, que al introducir la participación del médico en todo, limita el dominio de los individuos sobre su propio cuerpo ${ }^{22}$, transformando a la profesión médica en censora de la voluntad del individuo sobre estas materias.

Un tema menos abordado se refiere a la carga emocional que enfrentan los médicos al realizar el procedimiento. Como bien lo ejemplifica un estudio holandés sobre las opiniones de médicos que participan en MMA, "la persona que ayuda al paciente a morir tiene que seguir adelante con su vida, no el paciente"23. En este sentido, muchos médicos señalan sentirse presionados cuando reciben una solicitud de MMA y les cuesta encarar la carga emocional y la responsabilidad profesional de tener que tomar la decisión de aceptar o no dicha solicitud.

\section{Argumentos a favor de la participación de los médicos en la MMA}

La tradicional posición de las agrupaciones médicas, que sostienen que existe una moral interna, propia de la profesión, e independiente de las influencias externas y variaciones normativas que ocurran en la sociedad en que se desenvuelve, está siendo desafiada ${ }^{24}$. Para algunos bioeticistas, los fines de la medicina deben responder a un contrato social que atienda a los intereses de los pacientes y no a una supuesta moralidad propia de aquella, que se vería refutada por la variabilidad existente entre sus códigos deontológicos, en diferentes épocas y culturas ${ }^{20}$. Concordante con esto, se ha observado un cambio de opinión de los médicos que las conforman, como ha ocurrido al interior de la Asociación Médica Británica ${ }^{25}$ y en el COLMED. Un sondeo de opinión realizado el 2019 en Chile, el cual fue enviado a 24.013 médicos colegiados (22\% de respuestas) mostró que 59\% estaba dispuesto a administrar una droga letal a un paciente adulto competente que lo solicitare, si cumplía con requisitos bien estrictos ${ }^{26}$. La razón fundamental detrás de esta tendencia en la sociedad occidental es el reconocimiento de la autonomía de los individuos como un valor en sí mismo ${ }^{27}$. Según defensores de la eutanasia, esta cumpliría un rol benéfico en aquellos casos en que el sufrimiento ocasionado por la enfermedad se torna intolerable, incluso contando con acceso a los mejores cuidados paliativos. Para algunos filósofos morales, no existiría diferencia ética entre matar a un paciente que lo solicita o dejarlo morir luego de adecuar los esfuerzos terapéuticos. Esto último es hoy aceptado por la praxis médica, puesto que se reconoce la necesidad de suspender tratamientos considerados como fútiles, aun cuando de esta 
suspensión se siga la muerte del paciente ${ }^{28,29}$. En la mayoría de estos escenarios, los pacientes esperan la asistencia de un profesional médico, generando una tensión entre las expectativas de la comunidad respecto de la disposición del médico a atender aquella solicitud y la postura de las asociaciones médicas $^{30}$. En aquellos países donde es legal la MMA, se han establecido criterios específicos y una serie de procedimientos para garantizar una buena práctica. En Holanda se exige que se incluya el criterio de proporcionar el "cuidado debido", lo que significa que la petición sea voluntaria, esté bien informada, sea reiterada en el tiempo, que el paciente se encuentre con un sufrimiento intratable e insoportable, que no haya otro medio de solucionar su situación, que esté atestiguado al menos por otro médico independiente y que el procedimiento de poner fin a la vida sea médicamente adecuado ${ }^{31}$. En Bélgica, la eutanasia se contempla dentro de las prestaciones que el equipo de cuidados paliativos puede otorgar ${ }^{32,33}$.

Otro argumento que fundamenta la participación de los médicos en la MMA es que estos deben verificar que se cumplan los requisitos legales y también integrar las comisiones que revisan el cumplimiento de dicha normativa. En estos casos, aunque el profesional médico no participa directamente en el procedimiento, contribuye a que puedan realizarse según lo dispuesto por la legislación. Asimismo, las sustancias que se administran o prescriben deben asegurar que la muerte se produzca de una manera adecuada, sin ocasionar dolor ni otros síntomas, siendo el médico quien puede enfrentar de manera más competente eventuales complicaciones. Otras personas defensoras de la participación de la profesión médica aluden al principio de "no abandono" 34,35 , pues el médico, que ha seguido de cerca a su paciente durante el transcurso de su enfermedad, debe atender a su solicitud en caso de sufrimiento, entendiendo la muerte como un alivio y parte del cuidado integral al fin de la vida ${ }^{36}$. Una eutanasia que sigue fielmente los requisitos considerados por la ley, y que respeta los deseos y valores de las personas, es considerada como un acto profesional y compasivo.

Para quienes sostienen posturas favorables a la participación del médico en la MMA, esta no constituye relativismo moral o mera respuesta al cambio legislativo, sino que se origina de la constatación de diferencias en las valoraciones de la sociedad, las que finalmente motivan dichos cambios. En aquellos países en que se ha legalizado la MMA, nunca un médico es obligado a realizar el procedimiento, respetando así su derecho a invocar objeción de conciencia. El reconocimiento del derecho fundamental a la libertad de conciencia es expresión del pluralismo ético, tal como lo señalamos en nuestro artículo sobre el tema de la objeción de conciencia ${ }^{37}$.

Más allá de posturas favorables o contrarias a la MMA, existe discusión respecto de cuáles serían las tareas exclusivas que corresponden a la profesión médica. A modo de ejemplo, temas relacionados con diagnóstico, pronóstico y alternativas terapéuticas son claramente de resorte médico ${ }^{38}$. En cambio, temas espirituales y de manejo del sufrimiento, pueden ser mejor abordados por otras profesiones. Es así como en Suiza, la participación del médico se limita a la prescripción de la droga y certificación de que se cumple con los requisitos legales para acceder al procedimiento ${ }^{34}$. Al respecto, la Sociedad Europea de Cuidados Paliativos afirma que es responsabilidad de los profesionales de cuidados paliativos escuchar y explorar las solicitudes implícitas o explícitas para eutanasia, abordando el sufrimiento subyacente ${ }^{5}$.

El concepto de "dolor total" permite entender el proceso al final de la vida como una experiencia de enfermedad, padecimiento y disfunción social, para lo cual se requiere de una atención integral ${ }^{39}$. Sería reduccionista considerar que el profesional médico sólo se centre en el manejo de los síntomas físicos que aquejan a su paciente y no procure, mediante este cuidado integral, la mejor calidad de vida posible. Esto es concordante con una visión más inclusiva de los fines de la medicina que considera como responsabilidad de los médicos acompañar a sus pacientes en esta última etapa, en que deciden terminar voluntariamente con sus vidas, sin abandonarlos y proveyendo los medios necesarios para que esta decisión sea tomada cumpliendo todos los requisitos que la validan ética y legalmente ${ }^{10,34}$, a la vez que asegurando que el desenlace ocurra con el menor sufrimiento posible y la menor posibilidad de error ${ }^{40}$.

\section{Conclusiones}

Es imposible para la profesión médica eludir la responsabilidad que le cabe en la discusión acerca de la MMA y sobre la legitimidad o no de 
su participación en esta. Pese a la oposición de la mayor parte de las asociaciones médicas para participar de la MMA, legislaciones de diferentes tradiciones y latitudes siguen avanzando en el debate acerca del derecho a la muerte asistida. La mayoría de estas leyes considera al médico como el agente principal en la administración de estos medios, por considerar que se encuentra mejor calificado para garantizar que tengan la menor posibilidad de error, y validar que hayan sido satisfechos los requisitos acordados y sancionados legalmente. Una posición informada sobre esta materia requiere acceder, de modo equilibrado, a las diversas opiniones existentes, sin perder de vista que los intereses primarios del acto médico siguen siendo el bienestar del paciente y el alivio de su sufrimiento.

En síntesis, la participación del médico en la muerte de su paciente ha sido considerada por algunos como un acto fuera de los fines de la medicina, por lo que sería un acto no-médico. Otros la consideran como parte del quehacer clínico. En el presente documento, hemos querido representar las distintas miradas que existen al interior del Departamento de Ética del COLMED, para entregar así herramientas de reflexión a los colegas y estimular su participación en este debate.

\section{Referencias}

1. Almeyda R, Carvajal Y. Manuel Almeyda y el derecho a morir. Revista Chilena de Salud Pública 18.2 (2014): 206-9.

2. Brody BA. (Ed.). Suicide and euthanasia: historical and contemporary themes (Vol. 35). Dordrecht, Países Bajos: Spinger Science \& Business Media. 1989.

3. Cámara de Diputados, "Legislatura $366^{\mathrm{a}}$, Sesión $60^{\mathrm{a}}$ ", página 48, 21 de agosto de 2018. Disponible en: http:// www.camara.cl/pdf.aspx?prmID=13210 \&prmTIPO=TEXTOSESION (Fecha de acceso el 1 de agosto de 2019).

4. Simon Lorda P, Barrio Cantalejo IM, Alarcos Martínez FJ, Barbero Gutiérrez J, Couceiro A, Hernando Robles P. [Not Available]. Rev Calid Asist 2008; 23 (6): 271-85.

5. Radbruch L, Leget C, Bahr P, Muller-Busch C, Ellershaw J, de Conno F, et al. Euthanasia and physician-assisted suicide: A white paper from the European Association for Palliative Care. Palliat Med 2016; 30 (2): 104-16.

6. Beca JP, Ortiz A, Solar S. [The debate about the right to die]. Rev Med Chile 2005; 133 (5): 601-6.

7. Carrasco MV, Crispi F. [Euthanasia in Chile]. Rev Med
Chile 2016; 144 (12): 1598-604.

8. Goic A. [Notes on euthanasia]. Rev Med Chile 2005; 133 (3): 371-5.

9. Vivanco A. La autonomía de la persona frente al derecho a la vida no incluye el derecho a ser muerto por un tercero: La solicitud de asistencia al suicidio y el caso de Diane Pretty. Acta Bioethica 2002; 8 (2): 299-313.

10. Kottow M. Eutanasia y Res publica. Rev Chil Salud Pública 2005; 9 (2): 111-7.

11. Anderson JG, Caddell DP. Attitudes of medical professionals toward euthanasia. Soc Sci Med 1993; 37 (1): 105-14.

12. Dowbiggin, Ian. A concise history of euthanasia: life, death, God, and medicine. Lanham, MD: Rowman \& Littlefield, 2007.

13. Sjostrand M, Helgesson G, Eriksson S, Juth N. Autonomy-based arguments against physician-assisted suicide and euthanasia: a critique. Med Health Care Philos 2013; 16 (2): 225-30.

14. Gevers S. Euthanasia: law and practice in The Netherlands. Br Med Bull 1996; 52 (2): 326-33.

15. Netherlands State Commission on E. Final report of the Netherlands State Commission on Euthanasia: an English summary. Bioethics 1987; 1 (2): 163-74.

16. Young J, Egan R, Walker S, Graham-DeMello A \& Jackson C (2019) The euthanasia debate: synthesising the evidence on New Zealander's attitudes, Kōtuitui: New Zealand Journal of Social Sciences Online, 14:1, 1-21, DOI: 10.1080/1177083X.2018.1532915

17. WMA-The World Medical Association-WMA Resolution on Euthanasia and Physician-assisted suicide. Adoptada por la 70 Asamblea General de la AMM, Tbilisi, Georgia, octubre 2019. Disponible en https://http:// www.wma.net/policies-post/declaration-on-euthanasia-and-physician-assisted-suicide/ (Fecha de acceso el 29 de noviembre de 2019).

18. Código de Ética 2011. Colegio Médico de Chile (AG). Editado e impreso por Ediciones Tierra Mía Ltda. Santiago de Chile.

19. Grupo de Estudios de Etica Clinica de la Sociedad Medica de S. [Euthanasia and medical act]. Rev Med Chile 2011; 139 (5): 642-54.

20. The goals of medicine. Setting new priorities. Hastings Cent Rep 1996; 26 (6): S1-27.

21. McLean, Sheila AM. Assisted dying: reflections on the need for law reform. Abingdon: Routledge-Cavendish, 2007.

22. Salem T. Physician-assisted suicide. Promoting autonomy-or medicalizing suicide? Hastings Cent Rep 1999; 29 (3): 30-6.

23. Dees MK, Vernooij-Dassen MJ, Dekkers WJ, Elwyn G, 
Vissers KC, van Weel C. Perspectives of decision-making in requests for euthanasia: a qualitative research among patients, relatives and treating physicians in the Netherlands. Palliat Med 2013; 27 (1): 27-37.

24. Pellegrino ED. The internal morality of clinical medicine: a paradigm for the ethics of the helping and healing professions. J Med Philos 2001; 26 (6): 559-79.

25. Davis J. Most UK doctors support assisted dying, a new poll shows: the BMA's opposition does not represent members. BMJ 2018; 360: k301.

26. Colegio Médico de Chile, "Encuesta de opinión acerca de la muerte asistida médicamente", 2019. Disponible en: http://www.colegiomedico.cl/wp-content/ uploads/2019/04/Resumen-Encuesta-Eutanasia.pdf (Fecha de acceso el 1 de agosto de 2019).

27. Emanuel EJ. Euthanasia. Historical, ethical, and empiric perspectives. Arch Intern Med 1994; 154 (17): 1890-901.

28. Figueroa GR. [To kill, let die and euthanasia in the bill of rights of patients and in the Chilean doctrine]. Rev Med Chile 2011; 139 (5): 655-9.

29. Rachels, J. Active and passive euthanasia. N Engl J Med. 1975; 292 (2): 78-80.

30. Emanuel EJ, Onwuteaka-Philipsen BD, Urwin JW, Cohen J. Attitudes and Practices of Euthanasia and Physician-Assisted Suicide in the United States, Canada, and Europe. JAMA 2016; 316 (1): 79-90.

31. Rietjens JA, van der Maas PJ, Onwuteaka-Philipsen BD, van Delden JJ, van der Heide A. Two Decades of Research on Euthanasia from the Netherlands. What Have We Learnt and What Questions Remain? J Bioeth Inq 2009; 6 (3): 271-83.

32. Dierickx S, Deliens L, Cohen J, Chambaere K. Involvement of palliative care in euthanasia practice in a context of legalized euthanasia: A population-based mortality follow-back study. Palliat Med 2018; 32 (1): 114-22.
33. Bernheim JL, Raus K. Euthanasia embedded in palliative care. Responses to essentialistic criticisms of the Belgian model of integral end-of-life care. J Med Ethics 2017; 43 (8): 489-94.

34. Gamondi C, Borasio GD, Oliver P, Preston N, Payne $\mathrm{S}$. Responses to assisted suicide requests: an interview study with Swiss palliative care physicians. BMJ Support Palliat Care 2019; 9 (1): e7.

35. Vanden Berghe P, Mullie A, Desmet M, Huysmans G. Assisted dying -the current situation in Flanders: euthanasia embedded in palliative care. European Journal of Palliative Care 2013; 20 (6): 266-72.

36. Bernheim JL, Distelmans W, Mullie A, Ashby MA. Questions and answers on the Belgian model of integral end-of-life care: experiment? Prototype?: "Eu-euthanasia": the close historical, and evidently synergistic, relationship between palliative care and euthanasia in Belgium: an interview with a doctor involved in the early development of both and two of his successors. J Bioeth Inq 2014; 11 (4): 507-29.

37. Salas SP, Besio M, Borquez Estefo G, Salinas RA, Valenzuela CY, Micolich C, et al. [Position paper from the Department of Ethics of the Chilean College of Physicians about conscientious objection]. Rev Med Chile 2016; 144 (3): 382-7.

38. Bosshard G, Broeckaert B, Clark D, Materstvedt LJ, Gordijn B, Muller-Busch HC. A role for doctors in assisted dying? An analysis of legal regulations and medical professional positions in six European countries. J Med Ethics 2008; 34 (1): 28-32.

39. López-Sánchez J, Rivera-Largacha S. Historia del concepto de dolor total y reflexiones sobre la humanización de la atención a pacientes terminales. Rev Cienc Salud 2018; 16 (2): 340-56.

40. Savulescu J, Radcliffe-Richards J. A right to be unconscious. Anaesthesia 2019; 74 (5): 557-9. 